\title{
Dialisi peritoneale: da marginalità a opportunità
}

\section{Roberto Corciulo}

\author{
Azienda Ospedaliero-Consorziale Policlinico, Divisione di Nefrologia, Dialisi e Trapianto, Università di Bari, Bari \\ Coordinatore GdS Dialisi Peritoneale della Società Italiana di Nefrologia
}

\begin{abstract}
Peritoneal dialysis: From marginality to opPortunity
Abstract. Although several studies have demonstrated the clinical and economic benefits of peritoneal dialysis (PD) compared to hemodialysis (HD), PD as the initial modality is offered at much less than HD patients, causing in Italy the current annual prevalence rate of less than $\mathbf{1 0 \%}$. Making PD the default transition to dialysis therapy not only has clinical benefits for patients, but also for healthcare systems and society. This approach requires a cultural shift in the nephrologist and national and regional health systems, but it is time to make this shift. This article summarizes the views on how to increase the utilization of PD to improve on clinical and economic management of patients with ESRD.
\end{abstract}

Key words: Cost, Economic analysis, Hemodialysis, Peritoneal dialysis, Health policy

Conflict of interest: None.

Financial support: None.

Accettato: 20 Gennaio 2014

Quando, nel Marzo del 2013, Marco Lombardi mi propose, a distanza di 12 anni dalla pubblicazione "Dialisi Peritoneale: dialisi marginale" sul Supplemento del Giornale di Tecniche Nefrologiche \& Dialitiche (S1 GennaioMarzo 2001), di rilanciare il quesito a tutti i colleghi che avevano risposto alla prima richiesta, fui subito d'accordo ed entusiasta dell'iniziativa. Infatti, dopo 12 anni, la DP rimane "marginale" in termini di diffusione e considerai utile la raccolta dei pareri dei nefrologi che già avevano avuto modo di esprimersi su tale tematica, per cercare di capire in cosa si è sbagliato in questi anni e cosa sia, invece, utile fare per una maggiore e più omogenea diffusione della tecnica sul territorio nazionale. Purtroppo, dopo 12 anni, tra i nefrologi che avrebbero potuto partecipare all'iniziativa, è venuto prematuramente a mancare, nel Giugno u.s., Amedeo De Vecchi, amico buono e leale e nefrologo competente e stimato: la decisione di intitolare l'iniziativa alla sua memoria fu presa da Marco e da me senza che ci fossimo sentiti. Anzi, questo evento ci responsabilizzò ancora di più, perché il tentativo era di raccogliere pareri e suggerimenti per stimolare il dibattito sulla DP e per ottimizzare e migliorare la cura del paziente dializzato, gli stessi obiettivi che aveva Amedeo.

Il dibattito è stato articolato, con interventi tutti molto puntuali, talvolta critici ma sempre propositivi, e che hanno individuato, con un'attenta definizione, tutte le cause di una difficile diffusione della DP oggi in Italia.
Dall'analisi dei vari contributi inviati, sono sempre più convinto che la DP più che "dialisi marginale" sia stata “dialisi marginalizzata" un po' da tutti gli attori coinvolti nella proposta e nella gestione clinica ed economica.

La DP è ancora la "Cenerentola" dei trattamenti dialitici: è vero, ma Cenerentola non poteva andare al ballo perché qualcuno non voleva che ci andasse, come giustamente suggeriva un collega nel suo intervento!

Da un'analisi di quanto è successo e succede tuttora in Italia, si può affermare che la DP è stata marginalizzata da quelle equipe sanitarie che:

- non hanno avuto alcun interesse a conoscerla, proporla o imparare a gestirla

- hanno un primario che "non crede", quasi fosse una religione, nella DP e, pertanto, sono state ostacolate nell'utilizzarla e, pur avendo acquisito adeguate conoscenze per gestirla, non "credono" nella DP e, pertanto, pensano che, per il bene del paziente, sia opportuno non proporla e che, di conseguenza, non sia utile imparare a gestirla

- "credono" nella DP ma non hanno i mezzi o il personale necessario per proporla, pur sapendo gestirla

- riferiscono di non poterla utilizzare perché hanno prevalentemente pazienti di età avanzata e non autosufficienti, anche se, però, non la utilizzano nemmeno nei pazienti giovani e autosufficienti

- trattano pazienti al loro domicilio ma non hanno il "giusto know-how" per gestire la metodica per scarsa esperienza o 
scarsa conoscenza (spesso gestiscono pochi pazienti)

- non gestiscono il trattamento in maniera prudente e non seguono Best Practice e Linee Guida per il follow up clinico del paziente

- pensano che sia il miglior trattamento possibile e lo propongono a tutti i pazienti, anche se non hanno le giuste indicazioni

Inoltre, è stata marginalizzata anche da quei medici di medicina di base che:

- non conoscono la DP o non ne conoscono i benefici e i rischi e, quindi, dissuadono dal praticarla i pazienti che chiedono loro consigli

-pensano che gestire al domicilio un paziente in dialisi possa investirli di responsabilità e/o di impegni di cui non possono caricarsi.

Ma la DP è stata marginalizzata soprattutto da quelle istituzioni sanitarie (Ministero della Salute e Regioni) che non sono state mai attente a programmare la spesa sanitaria considerando qualità delle cure erogate e ottimizzazione delle risorse economiche disponibili.

Nel Marzo del 2009 (1), il Censis ha pubblicato i risultati di un'indagine sugli aspetti clinici, sociali ed economici dei trattamenti dialitici in Italia. L'indagine ha ribadito che, almeno per i primi anni di trattamento, DP e HD sono equivalenti per sopravvivenza del paziente; il Registro Europeo di Dialisi e Trapianto riporta una sopravvivenza simile dei pazienti nelle due metodiche fino al quinto anno di trattamento, salvo nei pazienti diabetici, in cui i risultati sono più sfavorevoli per i pazienti in DP, e il Registro Italiano di Dialisi e Trapianto indica una migliore sopravvivenza dei pazienti in DP nei primi tre anni di trattamento e una non significativa differenza nei tre anni successivi. Il Registro Italiano ha confermato che la DP rispetto all'HD preserva per più tempo la funzione renale residua che è fattore di migliore sopravvivenza sia nei pazienti in DP che in quelli in HD. Infine la DP, in corso di trapianto, si associa a una ripresa funzionale più precoce del rene trapiantato rispetto ai pazienti in HD, anche perché spesso i pazienti sono più giovani e in condizioni cliniche migliori. Lo stesso studio Censis ha, inoltre, confermato che la DP è in grado di garantire una migliore qualità di vita al paziente in dialisi. Quando sono stati confrontati gli aspetti economici, la DP, nella metodica automatizzata o manuale, è risultata più favorevole, come costi economici (rispettivamente da $-8.5 \%$ a $-27 \%$ ), rispetto all'emodialisi ospedaliera con bicarbonato e ancora di più quando è stata considerata la somma dei costi economici e di quelli sociali (rispettivamente da $-52 \%$ a $-19.7 \%$ ). Da questa indagine è risultato che il costo economico (costi diretti + costi indiretti) del trattamento di DP manuale è di $€ 25.520$, che quello automatizzato è di $€ 31.860$, che quello della bicarbonato dialisi ospedaliera è di $€ 34.827$ e, infine, che quello dell'HDF è di $€ 41.943$ all'anno. Recentemente, il Censis ha condotto uno studio (2), realizzato in collaborazione con la Società italiana di Nefrologia e con il Centro nazionale trapianti, sui costi economici del trapianto di rene rapportati a quelli della dialisi e ha evidenziato che il costo medio economico complessivo di un paziente trapiantato è di $€ 95.247$ in un periodo di tre anni. Il costo del trapianto in tre anni è pari a circa il $29.2 \%$ in meno rispetto a quello della dialisi, che è, invece, di $€ 123.081$. Il costo della dialisi nei tre anni è stato calcolato come media ponderata di tutti i tipi di trattamento dialitico (HD, HDF, APD, CAPD), ai quali sono stati aggiunti i costi relativi ai ricoveri, alla terapia farmacologica, alle prestazioni ambulatoriali e ai decessi. Se si considerano, invece, i costi di un paziente in DP (media ponderata tra APD e CAPD) che, in base al precedente lavoro del Censis, ammontano, per un periodo di tre anni, a circa $€ 99.114$, si evince che il costo del trapianto in tre anni è quasi coincidente con il costo di un paziente in DP nello stesso periodo. Quasi la metà è rappresentata dai costi sociali a carico dei pazienti trapiantati e dei loro familiari rispetto ai pazienti dializzati (€ 22.781 vs $€ 42.805$ ); se, invece, si considerano i costi sociali dei pazienti in DP, i costi sociali sono sovrapponibili a quelli dei pazienti trapiantati ( $€ 22.781$ vs $€ 22.588$ ).

Il trapianto renale, per $i$ vantaggi sia per il paziente (sopravvivenza, morbilità, qualità della vita) che per la società (riabilitazione del paziente, minori costi di gestione), è la migliore terapia dell'uremia terminale e compito principale della classe medica e delle istituzioni è di promuovere sempre questa modalità terapeutica, quando possibile. Recentemente è stato pubblicato il Report del Registro Veneto di Dialisi e Trapianto (3), che ha evidenziato una prevalenza dell'HD del 52\%, della DP del 10\% e del Tx del 38\%. Questa Regione si distingue dalla media nazionale per una consistente quota di pazienti trapiantati e una buona penetrazione della DP come trattamento dialitico (16.2\%). Ora, dal Report risulta che, dei pazienti in DP, il $6 \%$ riceve il Tx entro il primo anno dall'inizio del trattamento e il $34 \%$ entro i cinque anni. Dei pazienti in HD, invece, solo il $2 \%$ riceve il Tx nel primo anno di trattamento e solo il $16 \%$ entro i cinque anni. Nel totale dei pazienti in DP, il Tx è percentualmente la prima causa di drop out (12\% all'anno). Se si considera il fatto che il tempo medio di attesa per un trapianto di rene, nei pazienti in dialisi trapiantabili, è di circa tre anni, si può affermare che la sequenza terapeutica DP-Tx è quella che garantisce, nell'arco di sei anni di osservazione, la migliore sopravvivenza e la migliore qualità di vita con il costo (economico e sociale) totale più basso, rispetto allo stesso periodo di emodialisi. Inoltre, il costo (economico e sociale) del primo periodo in DP è sovrapponibile al secondo periodo con trapianto.

Nonostante queste valutazioni economiche, già note da tempo, non si è mai perseguita in Italia una politica di vera promozione della DP che potesse aumentare la bassa penetrazione nazionale di questa metodica che, oggi, è assestata attorno al $9-10 \%$.

Perché la DP non sia più marginalizzata, è necessario uno sforzo congiunto da parte di tutti gli attori coinvolti che "devono" assumersi la responsabilità, ognuno per la sua competenza, di portare avanti un programma di diffusione e promozione per migliorare la qualità di vita del paziente e, nel contempo, per ottimizzare le risorse umane ed economiche. Lo sforzo di una sola componente, che ritengo debba essere in primis quella istituzionale (ministeriale e regionale) sarebbe vano se non fosse accompagnato dalla 
partecipazione di intenti da parte degli altri attori e, pertanto, il progetto, che deve avere le caratteristiche di un "patto comune" finalizzato per raggiungere risultati stabiliti, non può che essere condiviso, costantemente verificato e settato a seconda dei risultati ottenuti. Il fallimento del progetto, per il venir meno di una delle parti coinvolte, lascerebbe la situazione allo stato attuale con "volontari" che si assumono "l'onere etico e professionale" di rispondere con più completezza alle esigenze dei propri pazienti e con l'indifferenza di chi, invece, dovrebbe, per compito istituzionale, organizzare e controllare l'assistenza e la spesa pubblica. In questo contesto, ritengo che il compito sinergico della SIN e del GdS di DP debba essere quello di sensibilizzare tutte le parti in gioco intervenendo sugli organi istituzionali e sulla classe medica (nefrologica e della medicina di base).

\section{Quali gli interventi da promuovere:}

SENSIBILIZZARE LE ISTITUZIONI SANITARIE SUL FATTO CHE LA SPESA SANITARIA NON SI DEVE "RAZIONARE" MA SI PUÒ "RAZIONALIZZARE". Non si può intervenire in sanità con tagli lineari ma si deve procedere con interventi mirati per ottimizzare la spesa e migliorare gli outcome clinici.

SENSIBILIZZARE LE ISTITUZIONI SANITARIE SULL'EVIDENZA CHE I TRATTAMENTI DIALITICI DOMICILIARI SONO PIÙ FAVOREVOLI IN TERMINI DI SPESA SANITARIA, PUR SENZA RINUNCIARE ALLA QUALITÀ DELLE PRESTAZIONI. L'utilizzo della DP potrebbe essere incrementato superando alcune criticità, tra le quali la più importante è la miopia della politica sanitaria vigente: Regione, Aziende Sanitarie e Aziende Ospedaliere non sembrano capaci di organizzare una sintesi che consideri costo complessivo del trattamento del paziente uremico (materiale per dialisi, personale sanitario e non, strutture dedicate, spesa farmaceutica, ospedalizzazioni, trasporti e costi sociali) e capacità di raggiungere l'obiettivo primario di cura (cioè sopravvivenza, riabilitazione e qualità di vita del paziente). In un simile confronto, la DP è il tipo di dialisi a minor costo a parità di efficacia con gli altri trattamenti.

Recentemente, sono stati pubblicati, in letteratura scientifica, numerosi lavori che affrontano il problema delle risorse economiche utilizzate nei trattamenti dialitici. In Europa, negli Stati Uniti e in Canada si è affermato con certezza che la DP è il trattamento più favorevole dal punto di vista dei costi economici e con il migliore rapporto costo-utilità. (4-7). Nazioni con prodotto interno lordo (PIL) PPA procapite elevato, cioè che tiene conto del PIL adeguato alle parità dei poteri di acquisto (8), come Stati Uniti ( $6^{\circ}$ posto), Hong Kong ( $7^{\circ}$ posto), Canada $\left(9^{\circ}\right.$ posto), Austria ( $11^{\circ}$ posto), Belgio ( $19^{\circ}$ posto), Regno Unito $\left(21^{\circ}\right.$ posto $)$, Francia $\left(23^{\circ}\right.$ posto $)$, Finlandia $\left(24^{\circ}\right.$ posto $)$ e Spagna $\left(29^{\circ}\right.$ posto $)$, stanno affrontando il problema dell'elevato impegno di risorse economiche necessarie per garantire $\mathrm{i}$ trattamenti dialitici e hanno già adottato (Hong Kong) o stanno per adottare la politica della "PD first" (9-11), cioè della DP come prima opzione terapeutica da offrire al paziente.

Pertanto, in tali realtà, è molto sentita la programmazione della spesa sanitaria e forte è l'interesse per promuovere, diffondere e incentivare questa metodica dialitica che, insieme al trapianto, rappresenta la migliore e, nel contempo, la più economica modalità di trattamento dell'uremia terminale.

L'Italia, che occupa, nella graduatoria mondiale del prodotto interno lordo (PIL) PPA procapite del 2012, il $30^{\circ}$ posto (-2 rispetto al 2011), nonostante la grave crisi economica e il grave deficit del bilancio dello Stato, con sempre minori risorse destinate al welfare, è ancora in forte ritardo su scelte strategiche in tal senso e nulla viene fatto a livello nazionale per promuovere la diffusione della DP.

É compito, pertanto, dei nefrologi e della SIN in particolare quello di sensibilizzare le istituzioni sanitarie nazionali e regionali per prendere decisioni che affrontino il problema della spesa sanitaria in campo dialitico con lungimiranza, efficacia ed efficienza.

La Società Italiana di Nefrologia dovrà interloquire con le istituzioni per definire strategie e pianificare interventi atti a promuovere una politica nazionale di maggiore diffusione della $D P$. Ciò comporterà la preparazione di percorsi diagnostico-terapeutici assistenziali (PDTA) sull'avvio del trattamento sostitutivo, con afferenze precoci al nefrologo e con l'istituzione di ambulatori per la malattia renale avanzata. Altro problema da affrontare sarà il rimborso del trattamento. Il confronto del costo/rimborso DP vs HD appare, nel 2012, più adeguato altrove (12) che non in Italia: un decreto ministeriale del Gennaio del 2013 (13) stabilisce rapporti monetari HD/DP che sono di 2.74 tra HD ospedaliera ospedaliera/APD contro 1.02 del Canada e 1.316 della Francia. Ciò imporrebbe, per una migliore diffusione della DP, un rimborso più equo e, quindi, più premiante per la DP. Altra possibilità di intervento è l'applicazione della quota unica capitaria per i trattamenti dialitici o, meglio, il metodo del "bundle reimbursement" adottato recentemente prima dal Portogallo e, quindi, dagli USA. In esso, una cifra forfettaria include la quasi totalità delle prestazioni necessarie, dialitiche, mediche, diagnostiche, terapeutiche, farmacologiche e nutrizionali, e garantisce per legge il pieno rimborso solo con il raggiungimento di target definiti. I risultati ottenuti dove sono stati applicati sono stati giudicati finora assai positivi $(14,15)$. Una maggiore e più partecipata strategia economica della SIN con le istituzioni sanitarie nazionali e regionali porterebbe, peraltro, al vantaggio di una condivisa ottimizzazione delle risorse destinate alla nefrologia piuttosto che a subire scelte di tagli lineari alla spesa.

Un programma di più diffusa domiciliarizzazione dei trattamenti dialitici comporta uno sforzo organizzativo, di informazione e di formazione che, però, non si può improvvisare. La fase di start-up del programma necessiterà di investimenti iniziali da destinare alla formazione della classe nefrologica, ma anche a sostenere l'assistenza domiciliare dei pazienti non autosufficienti. Il contesto familiare sempre più disgregato e individualista della società postmoderna non favorisce una metodica domiciliare in pazienti spesso non autosufficienti o al limite dell'autosufficienza. Infatti, il carico di lavoro e la responsabilità per le famiglie 
che si accollano la terapia dialitica domiciliare sono rilevanti e possono avere dei risvolti significativi sull'economia familiare e sulla tenuta psicologica dei membri della famiglia. Modalità di rimborso per le famiglie dei pazienti non autosufficienti in tutte le Regioni, sul modello della Regione Piemonte, potrebbero aiutare l'inserimento in DP di molti pazienti anziani o incapaci di eseguire da soli la DP o si potrebbe optare per forme di assistenza infermieristica domiciliare che appoggino la famiglia o che si sostituiscano alla famiglia nella gestione del paziente domiciliarizzato. Solo professionisti dedicati, motivati e capaci di seguire i pazienti a domicilio, in tutte le loro problematiche, possono, infatti, colmare parzialmente queste lacune e incoraggiare e sostenere i pazienti affinché ricevano al domicilio la migliore assistenza possibile. Esperienze di DP assistita sono già operanti in Nazioni europee come la Francia e hanno promosso la diffusione della DP in oltre 1' $80 \%$ dei pazienti di età superiore ai 75 anni non autosufficienti (16). Tali impegni economici potrebbero, peraltro, essere sostenuti investendo parte del risparmio generato dal suo minor costo rispetto all'emodialisi.

GARANTIRE SU TUTTO IL TERRITORIO LA PRESENZA DI EQUIPE NEFROLOGICHE PREPARATE A GESTIRE CON COMPETENZA LA DP. Ancora oggi, sul territorio nazionale, la DP è un'opzione terapeutica spesso non proposta ai pazienti o un'opportunità negata. Infatti, la DP è diffusa in maniera molto disomogenea tra nord e sud e nell'ambito della stessa Regione. Un centro dialisi su tre non la propone come opzione terapeutica. Il 60-65\% dei centri dialisi italiani la propone, ma solo il $30-35 \%$ la pratica con esperienza idonea e con risultati clinici apprezzabili. Pertanto, i centri che effettuano la DP con esperienza comprovata, in base al numero dei pazienti trattati, non sono più di un quarto di tutti i centri dialisi italiani.

Per ottenere buoni risultati occorre promuovere progetti di formazione e informazione ai nefrologi e ai medici di base. Le azioni strategiche da intraprendere sono:

- la diffusione della conoscenza della DP tra tutte le equipe nefrologiche impegnate nel trattamento dell'uremia con percorsi formativi personalizzati e tutoraggio residenziale. La scarsa conoscenza della DP come fattore marginalizzante della metodica è stata messa in evidenza anche dal censimento italiano sulla DP del 2006, che evidenzia come la DP venga effettuata nei centri in cui la componente medica ha avuto o ha esperienza nella metodica e come, invece, non venga praticata nei centri dove i nefrologi non hanno mai avuto esperienze in tale trattamento. Queste equipe non sono disponibili ad avviare una fase di start up che, dal punto di vista organizzativo e di impiego di risorse umane, è molto impegnativa e, di fatto, non sono in grado di offrire tutte le opzioni terapeutiche dialitiche disponibili. La promozione e la diffusione della DP nei centri a basso "know-how" possono essere sostenute da interventi di formazione con Format già rodati (percorso formativo programmato con moduli schedulati per il personale medico e infermieristico) e di affiancamento e tutoraggio da parte di una Struttura di Riferimento;

- la creazione di una rete di professionalità che aiuti tutte le realtà territoriali a superare le difficoltà logistiche, di conoscenza e gestionali. Fondamentali per una maggiore penetranza della DP sono: invio precoce al nefrologo, percorso informativo adeguatamente strutturato e disponibilità e conoscenza di tutte le opzioni di trattamento dell'IRC. Le opzioni terapeutiche che dovrebbero essere offerte al paziente con uremia terminale dovrebbero comprendere il trapianto da vivente o cadavere (singolo o doppio), pre-emptive o successivo, l'HD in centro, CAL o domiciliare, la CAPD e l'APD, a domicilio o presso RSA, la DP Intermittente (IPD) ospedaliera (anche se oggi ha indicazioni limitate) e il trattamento conservativo a oltranza.Un singolo centro difficilmente può offrire tutte queste opzioni che, tuttavia, tutte, vanno garantite al paziente; va, quindi, costituita una rete fra le diverse realtà nefrologiche. Esperti possono essere chiamati a costituire un nucleo di intervento territoriale (formato da medici e infermieri), capace di affiancarsi alle varie realtà che operano sul territorio, di individuare le criticità dei centri meno esperti (cliniche, chirurgiche e di reperimento dei pazienti idonei) e di predisporre piani di lavoro condivisi per aiutarle a risolvere problemi clinici e/o organizzativi che ne ostacolano l'attività;

- investire sulla formazione dei giovani nefrologi. In molte Università italiane, la specialità di Nefrologia non annovera la DP come insegnamento e, quindi, preclude una preparazione istituzionale completa per i nefrologi del futuro. È importante che la preparazione dei giovani nefrologi sia completa e che anche le Università sprovviste di tali insegnamenti si preoccupino di completare l'iter formativo con moduli educazionali diversificati per anno di corso;

- il coinvolgimento dei medici di medicina di base in un percorso di informazione su tutte le modalità di trattamento dell'uremia. La gestione consapevole e condivisa con il proprio paziente è un requisito fondamentale per una scelta partecipata del trattamento dialitico che più si addice alle esigenze cliniche e personali.

Intervenire con convinzione e fermezza sulle proposte operative succitate potrà consentire di liberare la DP dalla marginalità in cui è oggi relegata in Italia. Proporla come prima opzione terapeutica dialitica, con tutti i limiti che la tecnica deve avere, non produrrà vantaggi clinici solo per $\mathrm{i}$ pazienti, ma anche per il sistema sanitario e per la società. Questo approccio richiederà, però, un cambiamento culturale del nefrologo, del Sistema Sanitario Nazionale e dei sistemi regionali, ma adesso è il momento di fare questo cambiamento! ̇̀, questa, un'opportunità che penso non si possa disattendere per la particolare situazione della sanità del nostro Paese e per garantire, anche nei prossimi anni, la sostenibilità economica dei trattamenti dialitici a tutti i cittadini. Un'attenta gestione della spesa sanitaria in campo dialitico, con una terapia efficace ed efficiente, ci consentirà di operare al passo di Paesi ricchi perché lungimiranti e non ricchi e lungimiranti. 


\section{Riassunto}

Sebbene numerosi studi abbiano dimostrato i vantaggi economici e clinici della dialisi peritoneale (DP) rispetto all'emodialisi (HD), la DP come modalità iniziale di trattamento dialitico viene offerta molto meno rispetto all'HD e ciò è causa, in Italia, dell'attuale tasso di prevalenza annua inferiore al 10\%. Rendere la DP la prima opzione terapeutica dialitica non ha vantaggi clinici solo per i pazienti, ma anche per il sistema sanitario e per la società. Questo approccio richiede un cambiamento culturale del nefrologo, del Sistema Sanitario Nazionale e dei sistemi regionali, ma è il momento di fare questo cambiamento. Questo articolo riassume i punti di vista su come aumentare l'utilizzo della DP per migliorare la gestione clinica ed economica dei pazienti con uremia terminale.
Parole chiave: Costi, Analisi economica, Emodialisi, Dialisi peritoneale, Politica sanitaria

Dichiarazione di conflitto di interessi: L'Autore dichiara di non avere conflitto di interessi.

Contributi economici degli autori: L'Autore dichiara di non aver ricevuto sponsorizzazioni economiche per la preparazione dell'articolo.

Indirizzo degli Autori:

Roberto Corciulo

Divisione di Nefrologia, Dialisi e Trapianto

Azienda Ospedaliero Consorziale Policlinico

Università degli Studi di Bari

P.zza G. Cesare 11

70100 Bari

r.corciulo51@gmail.com

\section{Bibliografia}

1. I trattamenti sostitutivi della funzione renale in Italia: aspetti clinici, economici e sociali. Censis Marzo 2009.

2. Il valore del trapianto: un'analisi empirica dei consumi sanitari e dei costi dei trapiantati di rene in Italia. Censis Dicembre 2013.

3. Nordio M, Tessitore N, Feriani M, et al. Prevalence of renal replacement therapy in Veneto for 2008, 2009 and 2010. J Nephrol 2013; 26 (Suppl. 20): S13-22.

4. Coentrão L, Araújo CA, Ribeiro CA, Dias CC, Pestana MJ. Cost analysis of hemodialysis and peritoneal dialysis access costs in incident dialysis patients. Perit Dial Int 2013; 33 (6): 662-70.

5. Villa G, Rodriguez-Carmona A, Fernandez-Ortiz L, et al. Cost analysis of the Spanish renal replacement therapy programme. Nephrol Dial Transplant 2011; 26: 3709-14.

6. Neil N, Guest S, Wong L, et al. The financial implications for Medicare of greater use of peritoneal dialysis. Clin Ther 2009; 31 (4): 880-8.

7. Berger A, Edelsberg J, Inglese GW, Bhattacharyya SK, Oster G. Cost Comparison of Peritoneal Dialysis Versus Hemodialysis in End-Stage Renal Disease. Am J Manag Care 2009; 15 (8): 509-18.

8. http://it.wikipedia.org/wiki/Lista_di_stati_per_PIL_(PPA)_procapite.
9. Yu AW, Chau KF, Ho YW, Li PK. Development of the "peritoneal dialysis first" model in Hong Kong. Perit Dial Int 2007; 27 (Suppl. 2): S53-5.

10. Ghaffari A, Kalantar-Zadeh K, Lee J, Maddux F, Moran J, Nissenson A. PD First: Peritoneal Dialysis as the Default Transition to Dialysis Therapy. Semin Dial 2013; 26 (6): 706-13.

11. Chaudhary K, Sangha H, Khanna R. Peritoneal Dialysis First: Rationale. Clin J Am Soc Nephrol 2011; 6: 447-56.

12. Vanholder R, Davenport A, Hannedouche T, et al. Reimbursement of Dialysis: a Comparison of Seven Countries. J Am Soc Nephrol 2012; 23: 1291-8.

13. Supplemento ordinario n. 8 alla Gazzetta Ufficiale, Serie generale, n. 23, Prestazioni specialistiche ambulatoriali. 28-1-2013.

14. Ponce P, Marcelli D, Guerreiro A, et al. Converting to a capitation system for dialysis payment: the Portuguese experience. Blood Purif 2012; 34: 313-24.

15. Maddux FW. Impact of the bundled End-Stage Renal Disease payment system on patient care. Blood Purif 2012; 33: 107-11.

16. Castrale C, Evans D, Verger C, et al. Peritoneal dialysis in elderly patients: report from the French Peritoneal Dialysis Registry (RDPLF). Nephrol Dial Transplant 2010; 25: 255-62. 